\title{
Imaging the role of blood-brain barrier disruption in normal cognitive ageing
}

\author{
Inge C. M. Verheggen $(D \cdot$ Joost J. A. de Jong • Martin \\ P. J. van Boxtel • Alida A. Postma • Jacobus F. A. \\ Jansen • Frans R. J. Verhey • Walter H. Backes
}

Received: 21 July 2020 / Accepted: 28 September 2020 / Published online: 6 October 2020

(C) The Author(s) 2020

\begin{abstract}
To investigate whether blood-brain barrier (BBB) disruption is a potential mechanism of usual age-related cognitive decline, we conducted dynamic contrast-enhanced (DCE) MRI to measure BBB leakage in a healthy sample, and investigated the association with longitudinal cognitive decline. In a sample of neurologically and cognitively healthy, older individuals, BBB leakage rate in the white and grey matter and hippocampus was measured using DCE MRI with
\end{abstract}

I. C. M. Verheggen $(\bowtie) \cdot$ M. P. J. van Boxtel •

F. R. J. Verhey

Department of Psychiatry and Neuropsychology, Maastricht University, P.O. Box 616, 6200 MD Maastricht, The Netherlands e-mail: inge.verheggen@maastrichtuniversity.nl

I. C. M. Verheggen · J. J. A. de Jong • M. P. J. van Boxtel •

A. A. Postma · J. F. A. Jansen · F. R. J. Verhey •

W. H. Backes

School for Mental Health and Neuroscience (MHeNs), Maastricht University, Maastricht, The Netherlands

I. C. M. Verheggen • M. P. J. van Boxtel • F. R. J. Verhey

Alzheimer Center Limburg, Maastricht, The Netherlands

J. J. A. de Jong · A. A. Postma · J. F. A. Jansen ·

W. H. Backes

Department of Radiology and Nuclear Medicine, Maastricht

University Medical Center, Maastricht, The Netherlands

J. F. A. Jansen

Department of Electrical Engineering, Eindhoven University of Technology, Eindhoven, The Netherlands

W. H. Backes

School for Cardiovascular Research Institute Maastricht (CARIM), Maastricht University, Maastricht, The Netherlands pharmacokinetic modelling. Regression analysis was performed to investigate whether the leakage rate was associated with decline in cognitive performance (memory encoding, memory retrieval, executive functioning and processing speed) over 12 years. White and grey matter BBB leakages were significantly associated with decline in memory retrieval. No significant relations were found between hippocampal BBB leakage and cognitive performance. BBB disruption already being associated with usual cognitive ageing, supports that this neurovascular alteration is a possible explanation for the cognitive decline inherent to the ageing process. More insight into BBB leakage during the normal ageing process could improve estimation and interpretation of leakage rate in pathological conditions. The current results might also stimulate the search for strategies to maintain BBB integrity and help increase the proportion people experiencing successful ageing. Netherlands Trial Register number: NL6358, date of registration: 201703-24.

Keywords Blood-brain barrier - Cognitive ageing . Dynamic contrast-enhanced MRI · Cerebrovascular dysfunction $\cdot$ Successful ageing

\section{Introduction}

Over the last years, population demographics have been shifting towards a larger proportion of older individuals, due to a combination of decreasing birth rates and increasing life expectancy [1]. With a larger older 
population, determining which factors could lead towards successful ageing has become an important scientific objective even more. Besides the absence of disease and disability, an important component of successful ageing is the maintenance of cognitive functioning [2]. An ageing population is increasingly confronted with age-related neurobiological changes, and such changes in brain structure and function are often associated with changes in cognition $[3,4]$. However, ageing does not necessarily lead to cognitive impairment, as some people age without considerable cognitive setback, while others show substantial cognitive decline [5]. Investigating what neurobiological changes lead to age-related cognitive decline and hinder successful ageing is becoming increasingly important $[4,6]$.

Age has a large impact on the cerebrovascular system and is a major risk factor for cerebrovascular damage [7, 8]. Cerebrovascular disease is therefore a highly prevalent age-related condition [9]. Moreover, cerebrovascular disease appears to affect cognition even in elderly in the normal ageing spectrum [10]. The blood-brain barrier (BBB) is part of the cerebrovascular system and was shown to be vulnerable to age-related changes $[9,11$, 12]. Recently, higher BBB permeability has been linked to an increasing number of age-related neurological disorders $[8,13,14]$. Previous studies were even able to find an association between cognitive impairment inherent to these conditions and BBB disruption $[8$, 15-17].

At the level of the microvascular network, specialized cells work together in a neurovascular unit (NVU) to maintain cerebral blood flow (CBF), oxygen delivery and energy supply [18]. The NVU consists of vascular cells (i.e. endothelial cells), glial cells (i.e. astrocytes) and neurons $[18,19]$. Interaction between vascular cells and neurons in the NVU creates hemodynamic coupling, allowing an increase in neuronal activation to be followed by an appropriate CBF response [20, 21]. The endothelial cells are connected by tight junctions to create a strong barrier forming the basis of the BBB [18]. The BBB separates blood plasma from brain tissue and regulates the delivery of energy metabolites and nutrients to the neurons, while preventing neurotoxins from entering the brain.

The endothelium allows small molecules as oxygen and carbon dioxide to rapidly and freely diffuse across the $\mathrm{BBB}$, which is essential for brain metabolism and $\mathrm{pH}$ regulation [22]. Small, lipophilic molecules can also cross the BBB [23]. As for the rest, the endothelium contains specialized transport systems using active efflux, carrier-mediated transport and receptor-mediated transport to enable nutrients to pass from blood plasma to brain tissue and to remove waste products from the brain $[22,24]$. The BBB thereby protects the brain from ion fluctuations that occur in the blood after a meal or exercise and ensures homeostasis is maintained, which is essential for neuronal and synaptic functioning [25, 26].

Neurovascular dysfunction has been suggested to initiate the cascade leading to neurodegeneration and AD [18, 25, 27]. Neurovascular dysfunction involves uncoupling of the NVU, which can lead reduction and dysregulation of CBF and disruption of the BBB [28, 29]. A recent hypothesis for instance states that depletion of the nicotinamide adenine dinucleotide $\left(\mathrm{NAD}^{+}\right)$ substrate can lead to endothelial dysfunction, which impairs the integrity of the cerebral microcirculation [30]. Both CBF and BBB disruption impair the supply of oxygen and nutrients to the neurons. As a result, hypoxia and inflammation may occur and trigger subsequent pathological processes, which make the brain vulnerable to neuronal dysfunction, and eventually neurodegeneration [8, 31, 32]. Moreover, BBB disruption reduces the clearance of interstitial solutes from the brain and could lead to accumulation of toxic waste products, such as the amyloid- $\beta$ protein $(A \beta)$ in case of $\mathrm{AD}$ [33]. In turn, $\mathrm{A} \beta$ can further impair neurovascular functioning, including $\mathrm{CBF}$ regulation and BBB integrity. These neurovascular alterations occur even before the formation of $A \beta$ plaques, suggesting that they are an early event in the pathological cascade [34-36], which is supported by several imaging studies demonstrating early BBB disruption in the development of AD [15, 37-39]. Studies have even shown that carrying the $\varepsilon 4$ allelle of the apolipoprotein $\mathrm{E}$ gene (APOE4), indicating genetic susceptiblility to $\mathrm{AD}$ [40], is associated with accelerated BBB disruption [41-43].

Thus, BBB disruption is considered as an early event in the pathological cascade possibly leading to neurodegeneration $[15,38]$. Early BBB leakage was also associated with global cognitive functioning, as leakage rates in individuals with early $\mathrm{AD}$ and healthy agematched controls correlated significantly with cognitive function (Mini-Mental State Examination, MMSE [44]) [15]. Moreover, individuals with early cognitive dysfunction, not accounted for by a neurological disorder, demonstrated increased BBB disruption independent of 
$\mathrm{A} \beta$ or tau [17]. This suggests that BBB breakdown could already be present in those who experience agerelated cognitive decline, even without having a neurological disorder. A recent study demonstrated that BBB disruption is not only more prominent in APOE4 carriers, but this effect was stronger in cognitively impaired individuals, which demonstrates that BBB disruption is involved in APOE4-related cognitive decline [45]. BBB disruption might be a contributing mechanism to cognitive ageing.

Interest in BBB disruption is increasing due to recent discoveries about its role in brain pathology, but also due to recent advances made in the development of magnetic resonance imaging (MRI) techniques to measure permeability $[31,46]$. One of the most promising techniques is dynamic contrast-enhanced (DCE) MRI, which measures contrast agent leakage from the blood plasma to the brain interstitial space over time, and allows the detection of subtle leakage values, for instance in dementia [47]. Using DCE MRI has made it possible to detect increased BBB leakage in mild cognitive impairment (MCI), the transition stage to $\mathrm{AD}$ [16] and small vessel disease [48], the most important cause of vascular dementia [49]. Increased BBB leakage at older ages was even found in healthy individuals [50]. However, whether BBB leakage is associated with variation in usual age-related cognitive decline remains to be investigated.

The current study used dual-time resolution DCE MRI to investigate whether BBB breakdown is already associated with cognitive decline in cognitively and neurologically healthy, older individuals. We hypothesised that BBB leakage would be significantly associated with usual age-related cognitive decline, which would support BBB disruption as contributing mechanism to cognitive ageing.

\section{Methods}

Participants

Fifty-seven participants (mean age, 66 years; age range, 47-91 years) were recruited from the Maastricht Aging Study (MAAS) [51]. Cognitively and neurologically healthy participants were selected, based on the following criteria: MMSE [44] score $\geq 25$; Disabilities Assessment of Dementia (DAD [52]) score $\geq 90 \%$; as reported in their medical history: no diagnosis of dementia, prodromal dementia, MCI, or any other psychiatric or neurological condition, no large structural brain abnormalities, brain surgery or brain trauma, and no cognitive impairment due to substance abuse; no contraindications for MRI scanning or gadolinium-based contrast agent (sufficiently functioning kidneys with glomerular filtration rate $(\mathrm{eGFR})>30 \mathrm{~mL} / \mathrm{min})$.

Cognitive decline

Participants performed a short battery of standardized cognitive tests. Memory function was measured using the verbal learning test (VLT [53]), in which the immediate recall score gives an indication of short-term episodic memory and learning, and the delayed recall score is considered to be a measure of long-term episodic memory. Processing speed was measured with the letter-digit substitution test (LDST [54]). Executive functioning was measured with the Stroop colourword test [55], in which the interference score is considered to be a measure of inhibition.

Cognitive decline was calculated by subtracting the participants' current score from their previous score in the last measure of MAAS approximately 12 years ago, so that a larger difference score would correspond to more cognitive decline. The interference score of the Stroop test was obtained using the time participants needed to complete the task and was the only test in which a larger difference score indicated less decline. The difference score of Stroop interference was reversed by multiplying difference scores with -1 , so that a larger score would correspond to more cognitive decline.

\section{MRI acquisition}

Images were acquired on a 3-Tesla MRI scanner (Achieva TX, Philips Healthcare, Best, The Netherlands) with a 32-channel head coil. The imaging protocol included a 3D T1-weighted sequence for anatomical reference, a 3D T2-weighted fluid attenuation inversion recovery (FLAIR) sequence for brain segmentation and a dual-time resolution dynamic contrast-enhanced (DCE) sequence for leakage calculations.

The fast sequence of the dual-time resolution DCE MRI had a dynamic scan interval of $3.2 \mathrm{~s}$ and consisted of 29 volumes with a voxel size of $2 \times 2 \times 5 \mathrm{~mm}$. During the fast sequence, the gadolinium-containing contrast 
agent $\left(0.1 \mathrm{mmol} / \mathrm{kg}\right.$ gadobutrol, Gadavist ${ }^{\circledR}$, Bayer AG, Leverkusen, Germany) was injected in the antecubital vein (injection rate $3 \mathrm{~mL} / \mathrm{s}, 20 \mathrm{~mL}$ saline flush). After the rapid spread over the vasculature, a slow sequence was applied for leakage into the brain tissue. The slow sequence had a dynamic scan interval of $30.5 \mathrm{~s}$ and consisted of 30 volumes with a voxel size of $1 \times 1 \times$ $2 \mathrm{~mm}$. Conversion of tissue signal intensity to contrast agent concentration was enabled by T1-mapping.

Structural brain characteristics and segmentation

T2-FLAIR images were also used to obtain scores on the Fazekas scale [56] for white matter hyperintensity (WMH) load, and T1-weighted images to obtain scores on the global cortical atrophy (GCA) scale [57] and the medial temporal lobe atrophy (MTA) scale [58]. Categorical scores on these visual rating scales were assigned by experienced neuroradiologists (A.A.P and W.M.P.).

WMH volume $\left(\mathrm{cm}^{3}\right)$ was quantified using the inhouse developed, semi-automated segmentation tool GIANT [59] and corrected for intracranial volume, as volumetric measures may be confounded by their correlation with head size [60].

Automated brain segmentations were created using FreeSurfer software (version 6.0.0 [61]), after which these segmentations were visually checked and manually adjusted (I.C.M.V.). From the FreeSurfer output, cortical thickness averaged over the whole brain (mm) and hippocampal volume averaged over the hemispheres $\left(\mathrm{cm}^{3}\right)$ were obtained, after which hippocampal volume was corrected for intracranial volume. For our analyses, total white matter, total grey matter (cortical and deep grey matter and hippocampus) and the hippocampus separately were extracted to create tissue masks [61].

\section{DCE MRI processing}

Both the fast and slow DCE MRI were motion corrected and a reference image was created using the average of the pre-contrast images in each sequence. The fast DCE MRI data was co-registered on the slow DCE MRI, and the reference slow DCE MRI was registered on the T1-weighted images for anatomical reference. Ultimately, the inverse of the obtained transformation matrix was used to transform the T1-weighted data to slow DCE space.
An individual vascular input function (VIF) was obtained for each participant by manually (I.C.M.V.) selecting at least 20 voxels in the superior sagittal sinus. The tissue signal in the brain ROIs was converted to contrast concentration by assuming a linear relationship and using the tissue relaxation time from the $\mathrm{T}_{10}$-map, while the blood plasma signal in the VIF was converted to contrast concentration using in vitro data [62] (diluted $\mathrm{MnCl}_{2}$ stock solution with varying gadobutrol concentrations ranging from 1 to $40 \mathrm{mM}$ and baseline $\mathrm{T} 1$ relaxation time comparable to human blood).

\section{Pharmacokinetic model analysis}

Voxel-wise pharmacokinetic modelling was applied to the contrast agent concentration time-curves in brain tissue and blood plasma. The most parsimonious model still giving a proper fit to the data was used, namely the Patlak model, which assumes no reflux from the brain tissue back to the blood and has been demonstrated to be suitable for brain tissue [63].

Applying the Patlak model to the contrast concentration time-curves gave us an estimation of the $K_{i}$-parameter $\left(\mathrm{min}^{-1}\right)$ and the $v_{p}$-parameter for each voxel. The $K_{i}$ rate can be considered a measure of the permeability surface area product that indicates the leakage rate from the blood plasma to the brain tissue, and the $v_{p}$-parameter can be considered a measure of the blood plasma volume. Histograms of these parameters in the grey and white matter and hippocampus were created and corrected for noise [15], after which the mean $K_{i}$ and $v_{p}$ were calculated for each region. Due to the noise correction, mean $K_{i}$ per ROI generally has a lower value than the leakage rate of specific voxels (as depicted on overlays) as a relatively large number of voxels do not exhibit obvious leakage or leakage levels below the noise level.

\section{Statistics}

Among the cognitive scores, only Stroop interference was non-normally distributed. Difference score and current score of Stroop interference and the leakage rate and blood plasma volume parameters were cube-root transformed to obtain normal distributions. WMH volume and hippocampal volume were divided by intracranial volume to correct for head size [60] and logtransformed to obtain normal distributions. 
Multiple linear regression was performed, with cognitive decline as dependent variable and leakage rate as predictor, in turn for the white matter, grey matter and hippocampus separately, while correcting for age, sex and education. $G^{*}$ power software was used to calculate the required sample size [64]. We wanted to be able to detect a medium effect size $\left(f^{2}=0.15[65]\right)$, with an overall significance level of $5 \%$ and power set at a conventional $80 \%$. Power analysis for multiple linear regression using four predictors [66] demonstrated that 55 participants would be sufficient, and we eventually included 57 participants, taking into account the possibility that a few participants might have had to be excluded in the post-processing phase. The regression models were tested for each cognitive domain, resulting in a total of four times, and results were therefore corrected for multiple comparisons using the Benjamini-Hochberg procedure [67], with four tests and a false discovery rate (FDR) of $5 \%$. Additional analyses were performed using the most recent measure of cognitive performance instead of cognitive decline, and using the blood plasma volume instead of the leakage rate.

Post hoc analyses were conducted to investigate the influence of other common indicators of structural brain integrity, namely WMH volume, cortical thickness and hippocampal volume, and blood plasma volume. These variables were separately added to the regression analyses as potential confounders for the relation between BBB leakage and cognition. Also, the association between these brain health measures and cognitive decline was investigated, using multiple linear regression with cognitive decline as dependent variable and WMH volume, cortical thickness, hippocampal volume or blood plasma volume as predictor, correcting for age, sex and education.

All statistical analyses were performed with commercial software (SPSS, version 24.0, IBM Corp., Armonk, NY, USA).

\section{Results}

Multiple linear regression was used to investigate the association between BBB leakage rate (white matter, grey matter and hippocampus) and 12-year cognitive decline (short-term and long-term memory function, processing speed and executive functioning), in a usual ageing sample ( $n=57$, see Table 1 for sample characteristics).

\section{Cognitive decline}

A significant decrease in cognitive score was found for immediate recall $(p=.023)$, processing speed $(p<.001)$ and interference $(p<.001)$.

\section{The association between BBB leakage and cognitive decline}

Older age is significantly associated with higher BBB leakage in the white matter $(\beta=.306, p=.024)$ and grey matter $(\beta=.286, p=.035)$, as can be read in more detail in Verheggen et al. [50]. Upon investigating whether this higher leakage is also associated with more agerelated cognitive decline, we found a significant relation

Table 1 Participant $(n=57)$ characteristics

\begin{tabular}{|c|c|c|}
\hline & \multicolumn{2}{|c|}{$\begin{array}{l}\text { Mean (standard deviation)/percentage/ } \\
\text { median (25th-75th percentile) }\end{array}$} \\
\hline Age & $65.8(10.2)$ & \\
\hline$\%$ Male & 52.6 & \\
\hline$\%$ Level of education ${ }^{a}$ & $1 / 2 / 3$ & $15.8 / 54.4 / 29.8$ \\
\hline $\mathrm{MMSE}^{\mathrm{b}}$ & $29.0(28.0-30.0)$ & \\
\hline$\%$ WMH Fazekas ${ }^{\mathrm{c}}$ & $0 / 1 / 2 / 3$ & $5.3 / 70.2 / 12.3 / 12.3$ \\
\hline$\% \mathrm{GCA}^{\mathrm{d}}$ & $0 / 1 / 2 / 3$ & $19.3 / 50.9 / 24.6 / 5.3$ \\
\hline \multicolumn{3}{|l|}{$\%$ MTA $^{\mathrm{e}}$} \\
\hline Right hemisphere & $0 / 1 / 2 / 3 / 4$ & 78.9/17.5/3.5/0.0/0.0 \\
\hline Left hemisphere & $0 / 1 / 2 / 3 / 4$ & 78.9/19.3/1.8/0.0/0.0 \\
\hline
\end{tabular}

a Level of education: 1, at most primary or lower vocational education; 2 , secondary education; 3 , higher vocational or scientific education

${ }^{\mathrm{b}}$ Mini-Mental State Examination score [44] at 12-year follow-up: maximum score $=30$, cognitively normal $\geq 25$

${ }^{\mathrm{c}}$ Fazekas visual rating score of white matter hyperintensity load [56]: 0 = absent: none or a single punctuate WMH lesion; 1 , mild: multiple punctuate lesions; 2 , moderate: beginning of confluency of lesions; 3 , severe: large confluent lesions

${ }^{\mathrm{d}}$ Global cortical atrophy visual rating score [57]: 0, absent: normal volume/no ventricular enlargement; 1 , mild: opening of sulci/mild ventricular enlargement; 2, moderate: volume loss of gyri/ moderate ventricular enlargement; 3, severe: 'knife blade' atrophy/severe ventricular enlargement

${ }^{\mathrm{e}}$ Medial temporal lobe atrophy visual rating score [58]: 0, absent: no atrophy; 1 , marginal: only widening of choroid fissure; 2 , mild: also widening of temporal horn of lateral ventricle; 3 , moderate: moderate loss of hippocampal volume; 4 , severe: severe volume loss of hippocampus 
between BBB leakage in the white and grey matter and decline in delayed recall (Table 2; Fig. 1; Fig. 2), with higher BBB leakage rates being associated with a larger decrease in delayed recall score, while correcting for age, sex and education (white matter $\beta=.389$, $p=.006$; grey matter $\beta=.287, p=.044$ ). Out of the four cognitive domains, leakage rate had the strongest regression coefficients with delayed recall (Table 2).

After correction for multiple comparisons using the Benjamini-Hochberg procedure [67] (four tests, FDR $=.05$ ), only the association between decline in delayed recall and white matter leakage remained significant.

Multiple linear regression with BBB leakage in the white matter as dependent variable and age as predictor, while correcting for sex, gave an unstandardized regression coefficient $(\beta)$ of $1.2 \cdot 10^{-4}$. This suggests that being 10 years older equals an increase of 1.2 . $10^{-3}$ in the (cube-root transformed) white matter $K_{i}$ rate. The above reported regression with white matter BBB leakage as predictor of decline in delayed recall score (standardized $\beta=.39$ (Table 2), unstandardized $\beta=286$ ) can be used to calculate that this amount of leakage would correspond to a decline of 0.35 units $\left(286 \cdot\left(1.2 \cdot 10^{-3}\right)\right)$ on the VLT delayed recall score. Considering the baseline MAAS data from 1855 healthy participants [68], not taking BBB leakage into account, this decline in cognition would normally correspond to an increase of 4.7 years of age. Thus, over the 10-year period, white matter BBB leakage alone is estimated to be associated with 4.7 years of cognitive ageing.

Table 2 Median and interquartile range (IQR) of the leakage rates and mean and standard deviation (SD) of cognitive decline in each cognitive domain, and the standardized regression coefficient $(\beta)$
The association between BBB leakage and current cognitive score

No significant association was found between BBB leakage in the white or grey matter, or hippocampus, and the most recent cognitive score for each cognitive domain. However, the association between BBB leakage in the white and grey matter and delayed recall score approached significance (white matter $\beta=-.259$, $p=.055$; grey matter $\beta=-.264, p=.050$ ), showing a trend towards more BBB leakage being associated with a worse performance on the delayed recall test.

\section{The association between blood plasma volume and cognitive decline}

No significant association was found between blood plasma volume in the white matter, grey matter or hippocampus and decline in any of the cognitive domains (white matter $\beta \geq-.171, p \geq .19$; grey matter $\beta \geq$ - .209, $p \geq .11$; hippocampus $\beta \leq .139, p \geq .33$ ).

Please note that the main analyses, i.e. BBB leakage and 12-year cognitive decline, was tested and corrected for multiple comparisons. No correction for multiple comparisons was applied to the analyses between BBB leakage and current cognitive score, or the analyses between blood plasma volume and cognitive decline, as these were additional analyses that yielded no significant results, and multiple comparisons correction, to check whether a significant result remains significant after correction, was not needed.

between leakage rate and cognitive decline, corrected for age, sex and education

\begin{tabular}{|c|c|c|c|c|c|}
\hline & $\begin{array}{l}\text { Cognitive decline } \mathrm{e}^{\mathrm{a}} \\
\text { Mean }(\mathrm{SD})\end{array}$ & $\begin{array}{l}K_{i}\left(10^{-6} \cdot \min ^{-1}\right) \\
\text { Median }(\mathrm{IQR})\end{array}$ & $\begin{array}{l}\text { White matter } \\
1.1(0.5 ; 2.0)\end{array}$ & $\begin{array}{l}\text { Grey matter } \\
0.9(0.4 ; 1.6)\end{array}$ & $\begin{array}{l}\text { Hippocampus } \\
1.7(0.4 ; 4.2)\end{array}$ \\
\hline Immediate recall & $2.4(6.6)^{*}$ & & $\beta=.200$ & $\beta=.083$ & $\beta=.056$ \\
\hline Delayed recall & $0.3(2.9)$ & & $\beta=.389 *$ & $\beta=.287^{*}$ & $\beta=.175$ \\
\hline Processing speed & $5.1(4.4)^{*}$ & & $\beta=.075$ & $\beta=.069$ & $\beta=.038$ \\
\hline Interference $^{\mathrm{b}}$ & $13.5(11.0)^{*}$ & & $\beta=.176$ & $\beta=.150$ & $\beta=.054$ \\
\hline
\end{tabular}

\footnotetext{
${ }^{\text {a }}$ Score last MAAS measure - current score

${ }^{\mathrm{b}}$ Reversed by multiplying with -1

*Significant at $p<.05$
} 

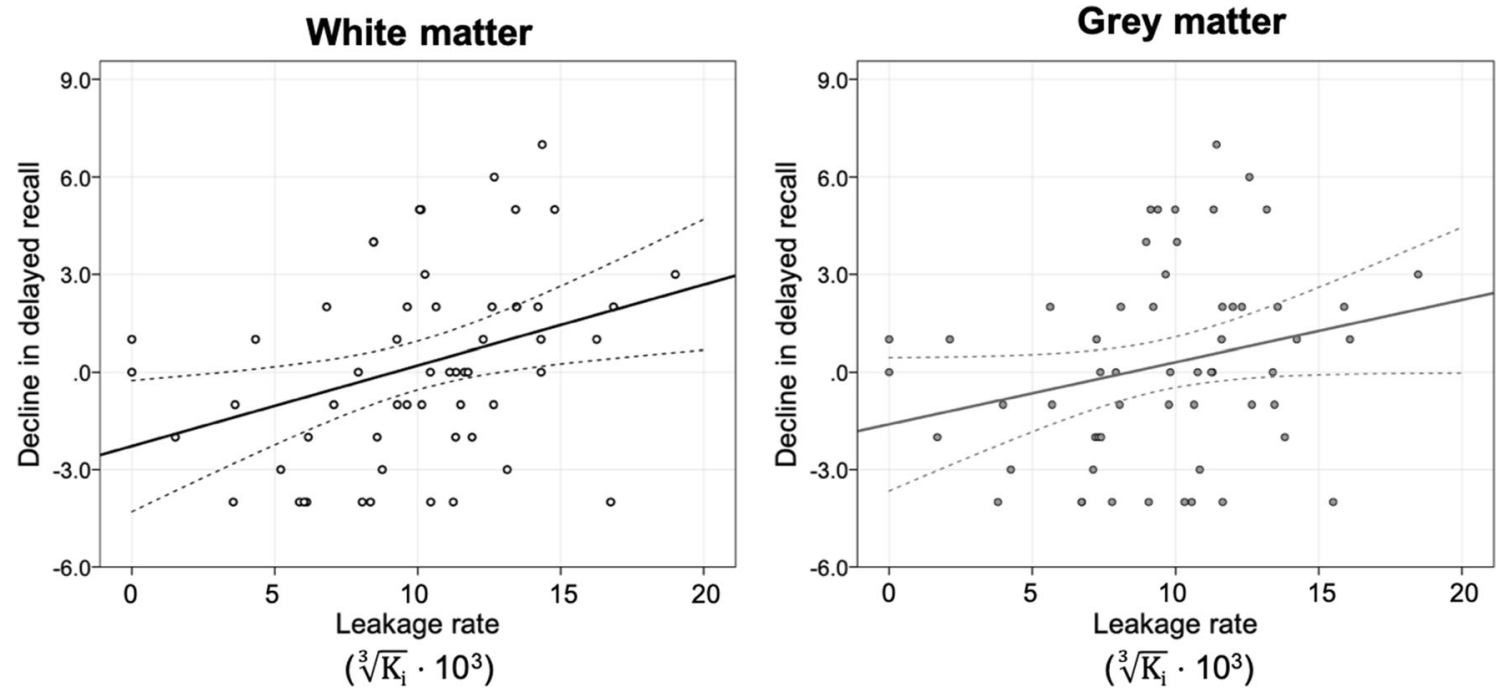

Fig. 1 Regression analysis demonstrated that white and grey matter leakage rate is significantly associated with decline in delayed recall $(n=57)$

The influence of WMH volume, cortical thickness, hippocampal volume and blood plasma volume

Post hoc analyses demonstrated that the association between BBB leakage in the white matter and decline in delayed recall remained significant after correction for WMH volume, cortical thickness, hippocampal volume or blood plasma volume. The association between grey matter BBB leakage and decline in delayed recall was just above significance when correcting for $\mathrm{WMH}$ volume $(p=.061)$, cortical thickness $(p=.064)$, hippocampal volume $(p=.066)$ or blood plasma volume $(p=.080)$. Results for the analyses with other ROIs or other cognitive domains did not change when adding WMH volume, cortical thickness, hippocampal volume or blood plasma volume to the regression analyses. None of these other MRI measures of structural brain integrity or blood plasma volume had a significant relation with cognitive decline.

\section{Discussion}

In the current study, we investigated whether BBB disruption is related to usual age-related cognitive decline. We found a significant association between BBB leakage and decrease in delayed recall score, which measures long-term episodic memory. Previous research has shown that decline in episodic memory is part of the normal ageing process $[69,70]$. Both acquisition (the ability to encode newly learned information into memory) and retrieval (the ability to access newly learned information) decrease across the lifespan. The delayed recall score is considered a measure of retrieval and we demonstrated that BBB disruption in the white matter was significantly associated with impairment in retrieval. The current study estimated that over a period of 10 years, white matter BBB leakage alone would be associated with 4.7 years of cognitive ageing. BBB disruption in the grey matter also showed an association with decline in retrieval, but this was less strong than in the white matter and no longer significant after correction for multiple comparisons.

The encoding of episodic events takes place in the hippocampus [71] first, and representations of these events are then stored in the cortex. For the retrieval function, both hippocampal and cortical processing is required with activation of a hippocampal-cortical network $[71,72]$. Especially pathology in the white matter tracts can disrupt communication between the hippocampus and cortex and may impair network function. This corresponds to our finding that decline in retrieval is strongly related to $\mathrm{BBB}$ disruption throughout the cerebral white matter, rather than specifically in the hippocampus. For future studies, it would be interesting to investigate what white matter tracts are involved and in which white matter regions the association between BBB leakage and cognitive decline is most prominent.

A previous study found a significant association between BBB leakage and age and demonstrated that BBB 

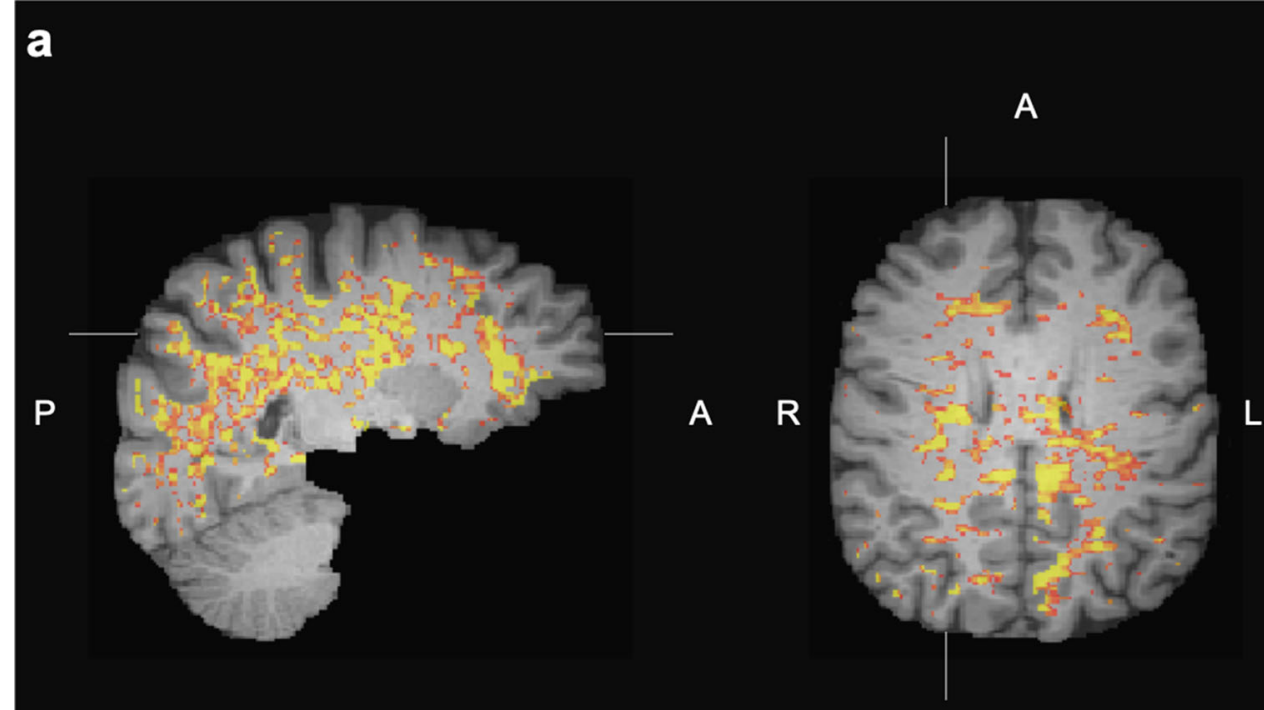

$\mathbf{P}$

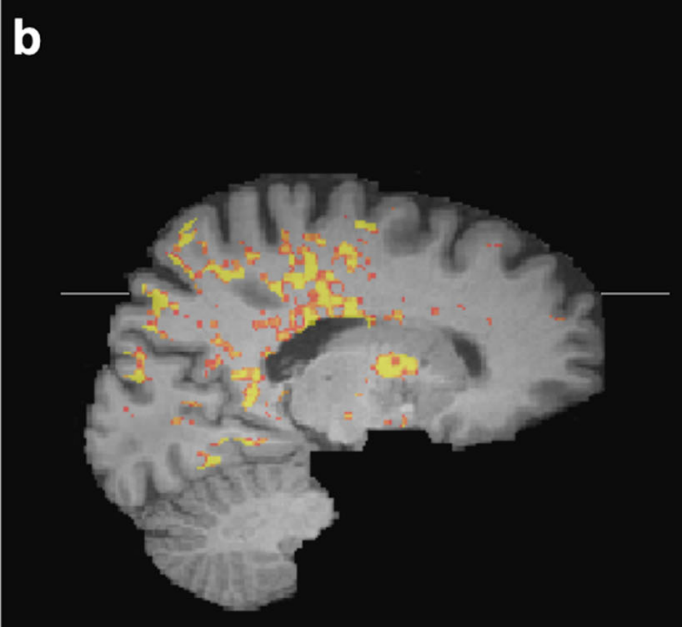

\section{Leakage rate $\mathrm{K}_{\mathrm{i}}\left(\mathrm{min}^{-1}\right)$}

\subsection{5}

0.001

Fig. 2 Leakage rate per voxel in the white matter of a a woman (57 years) with a large decrease in the delayed recall score (remembering seven words less) and $\mathbf{b}$ a man (62 years) with

leakage was significantly higher in individuals with mild cognitive impairment compared to normal controls [38]. Remarkably, these relations were only found for BBB leakage in the hippocampus, and not for leakage in white matter or cortical grey matter structures. The authors propose that, in normal ageing, BBB disruption possibly begins in the hippocampus and may subsequently contribute to age-related cognitive impairment. improved performance on the delayed recall test (remembering four words more)

However, while the current study demonstrated that BBB disruption was linked to usual age-related cognitive decline, we could only find this association for BBB disruption in the cerebral white matter, but not in the hippocampus. Thus, we were not able to find support for the previous suggestion that early BBB leakage beginning in the hippocampus contributes to cognitive impairment in normal ageing. 
In a later study, the same group found higher BBB leakage in the hippocampus of individuals with early cognitive dysfunction compared to healthy controls [17]. Possibly, the relation between BBB disruption in the hippocampus and cognitive performance is only present at a later stage of age-related cognitive deterioration. In another more recent study, they elaborate on this finding by demonstrating that cognitive impairment could especially be linked to BBB leakage in the hippocampus among APOE4 carriers [45]. As the APOE4 allele greatly increases the risk of a neurodegenerative disorder, especially AD [40], this indicates that BBB disruption in the hippocampus might be more involved in pathological ageing and might explain why the current study, focusing on successful ageing, obtained a different result. However, another previous study, not only investigating normal controls but also individuals with mild cognitive impairment and $\mathrm{AD}$, found no significant relation between BBB disruption in the hippocampus and cognitive performance [73]. The authors suggest that, at a later stage of neurodegeneration, the effect of microvascular damage on cognitive performance might be negligible compared to the effect of neurodegenerative processes. This study also did not find a significant relation between BBB disruption in the white or grey matter and memory performance. The discrepancy with our results could be due to the use of longitudinal cognitive data in our study. When using only the most recent measure of cognitive performance, we did not find a significant relation either, though a trend approaching significance was visible. This could indicate that the influence of BBB disruption on cognitive performance is best visible in the long term.

Performance on the cognitive tests is not only determined by cognitive ageing but also practice effects could influence the scores [74]. Especially memory scores are known to be susceptible to practice $[75,76]$. Cognitive decline could result from a combination of more severe cognitive ageing and less benefit from practice effects, and this combination can differ for each cognitive domain. Therefore, the current study did not aim to compare cognitive decline between the different domains. Instead, we investigated whether variation in a particular cognitive score was related to variation in leakage rate.

We did not find associations between BBB leakage and decline in immediate episodic memory as a measure of acquisition, processing speed, or the interference score as a measure of inhibition. As mentioned before, acquisition has been shown to decrease across the life span [69, 70]. Also, processing speed decreases with age, starting in the third decade $[69,77]$, and research has shown that inhibition is compromised in normal ageing, older adults $[78,79]$. Although each cognitive domain is thus known to be affected by age, only for decline in retrieval the association with BBB disruption reached significance. Previous imaging studies using diffusion tensor imaging (DTI) have suggested that usual age-related cognitive decline can at least partly be explained by white matter damage, with the greatest effect on cognitive functions that depend on communication between brain regions $[80,81]$. In a comparable sample of healthy, middle-aged to older individuals, no direct association could be found between white matter integrity, as measured with DTI, and processing speed or executive function [81]. However, white matter integrity was significantly associated with working memory, possibly because working memory involves communication between the medial temporal and the frontal system $[80,82]$. Already before these findings, working memory had been linked to early normal ageing, which was attributed to a disruption in balance between the two systems [83]. As mentioned above, retrieval also involves communication between medial temporal lobe structures and distributed cortical areas [71, 72], so this cognitive function could be most vulnerable to usual age-related decline, which would explain why we only found a significant relation with decline in retrieval.

After correction for WMH volume, cortical thickness, hippocampal volume or blood plasma volume, BBB disruption in the white matter remained significantly associated with decline in retrieval. Thus, the relation between BBB disruption and usual age-related cognitive decline could not be explained by other physiological ageing phenomena. BBB leakage being significantly associated with cognitive decline even after correction for blood plasma volume also indicates that the $K_{i}$ rate is not simply dependent upon capillary density. No extensive white matter lesion load, cortical thinning or hippocampal volume loss was present in the current study, as can be seen in the overall low Fazekas, GCA and MTA ratings (Table 1), and these variables were not associated with cognitive ageing. Also, while decreased vascularization has been associated with cognitive decline [84, 85], our study does not find any association between the blood plasma volume and cognition. However, BBB disruption was already associated with usual age-related cognitive decline at this stage, which 
supports the notion that BBB disruption is an early event in cognitive ageing, that might possibly affect brain function before these other processes.

To confirm our findings, longitudinal data should be collected to investigate whether BBB leakage increases as a person ages and whether this increase is paired with a change in cognitive function. Not only will a withinsubject longitudinal design have more power as the results are no longer influenced by between-subjects variation, it will also allow us to comment on causality and uncover whether BBB disruption precedes cognitive changes and other brain pathologies. With BBB leakage rate only being obtained at the end of the 12year interval, one cannot make inferences about causal relations, as persons with stronger BBB disruption and cognitive decline may have similar underlying origins. Neuroinflammation, for example, seems to form a vicious cycle with BBB disruption [86]. Defensins penetrate the $\mathrm{BBB}$ and trigger the release of inflammatory cytokines, which leads to BBB disruption and consequently access of defensins, $T$ cells and inflammatory mediators into the brain, further promoting neuroinflammation. Another example is cerebral small vessel disease (cSVD). cSVD burden was demonstrated to correlate with both stronger BBB disruption and smaller blood plasma volume [87]. Just like BBB disruption, neuroinflammation and cSVD have both been implicated in neurodegeneration and cognitive impairment [86, 88,89 ], and these processes may all be interacting with one another. It would have been preferable to measure BBB leakage at the moment of the first cognitive measure, so we would have been able to investigate whether baseline BBB disruption predicts future cognitive decline. However, 12 years ago, imaging techniques were not yet sensitive enough for the subtle leakage values in normal ageing, which rendered the current design the best alternative. Moreover, BBB leakage needs to be MRI detectable at these younger ages in similarly healthy persons.

Another point of consideration for this study was the difficulty in identifying usual age-related cognitive decline. Participants recruited from MAAS have been participating in research for over 25 years, and may represent an exceptionally healthy subgroup, who are likely to perform above-average for their respective age groups. Cognitive decline in normal ageing is subtle, especially in a sample this healthy. The standard cognitive tests, especially the memory scores, can lack sensitivity due to practice effects $[75,76]$, even in the current study with cognitive decline being calculated over a 12year interval. To minimize these effects, different versions of the VLT were used at the different time points (but fixed per time point), which have been demonstrated to be equivalent [90]. Moreover, this study benefitted from the use of the cognitive MAAS data [51]. Initial practice has the strongest effect [75], and we were able to use the third MAAS measure as our baseline to minimize practice effects.

\section{Conclusion}

BBB disruption throughout the cerebral white matter was associated with decline in retrieval in normal ageing. BBB breakdown being related to early cognitive decline supports the notion that BBB disruption could be a trigger of neuropathology eventually leading to neurodegeneration, and suggests that it could be a contributing mechanism to cognitive ageing. Future research should investigate the pathological sequence of events by using longitudinal imaging data. Moreover, cardiovascular risk and life-style factors could be relevant to study possible mitigation of the observed effect. Also, recent studies suggest that restoring $\mathrm{NAD}^{+}$levels can protect BBB integrity [91-93], and this effort might become relevant for the maintenance of cognitive function.

Acknowledgements The authors would like to acknowledge the participants for their contribution to scientific research, Walter M. Palm for neuroradiological assistance, Jos M.G.M. Slenter, Jan H. Jungerius and Ed H.B.M. Gronenschild for ongoing software, hardware and network support.

Author's contributions Inge C.M. Verheggen, MSc: conceptualization, data curation, formal analysis, funding acquisition, investigation, methodology, project administration, visualization, writing - original draft, writing - review and editing. Joost J.A. de Jong, PhD: data curation, formal analysis, methodology, software, visualization, writing - review and editing. Martin P.J. van Boxtel, MD, PhD: conceptualization, formal analysis, methodology, project administration, resources, supervision, writing - review and editing. Alida A. Postma, MD, PhD: data curation. Jacobus F.A. Jansen, PhD: conceptualization, formal analysis, methodology, visualization, writing - review and editing. Frans R.J. Verhey, MD, PhD: funding acquisition, methodology, project administration, resources, supervision. Walter $\mathrm{H}$. Backes, $\mathrm{PhD}$ : conceptualization, formal analysis, funding acquisition, methodology, resources, supervision, visualization, writing — review and editing. 
Funding This work was supported by the Dutch Research Council (NWO) (grant number 406-15-031).Data availabilityThe dataset supporting the current study has not been deposited in a public repository to keep participant data secure, but is available from the corresponding author on request.

\section{Compliance with ethical standards}

Conflict of interest The authors declare that they have no conflict of interest.

Ethics approval The study was approved beforehand by the medical ethics review committee of Maastricht University Medical Center (approval number: METC163050).

Consent to participate All participants provided written informed consent beforehand regarding participation.

Consent for publication All participants provided written informed consent beforehand regarding publication of the data.

Open Access This article is licensed under a Creative Commons Attribution 4.0 International License, which permits use, sharing, adaptation, distribution and reproduction in any medium or format, as long as you give appropriate credit to the original author(s) and the source, provide a link to the Creative Commons licence, and indicate if changes were made. The images or other third party material in this article are included in the article's Creative Commons licence, unless indicated otherwise in a credit line to the material. If material is not included in the article's Creative Commons licence and your intended use is not permitted by statutory regulation or exceeds the permitted use, you will need to obtain permission directly from the copyright holder. To view a copy of this licence, visit http://creativecommons.org/licenses/by/4.0/.

\section{References}

1. He W, Goodkind D, Kowal PR. An aging world: 2015. 2016.

2. Rowe JW, Kahn RL. Successful aging. The Gerontologist. 1997;37(4):433-40.

3. Glisky EL. Changes in cognitive function in human aging. Brain aging: models, methods, and mechanisms. 2007:3-20.

4. Rapp PR, Amaral DG. Individual differences in the cognitive and neurobiological consequences of normal aging. Trends Neurosci. 1992;15(9):340-5.

5. Cabeza R, Albert M, Belleville S, Craik FI, Duarte A, Grady $\mathrm{CL}$, et al. Maintenance, reserve and compensation: the cognitive neuroscience of healthy ageing. Nat Rev Neurosci. 2018;19(11):701-10.

6. Bishop NA, Lu T, Yankner BA. Neural mechanisms of ageing and cognitive decline. Nature. 2010;464(7288): 529-35.
7. Camici GG, Liberale L. Aging: the next cardiovascular disease? Eur Heart J. 2017;38(21):1621-3. https://doi. org/10.1093/eurheartj/ehx239.

8. Yang T, Sun Y, Lu Z, Leak RK, Zhang F. The impact of cerebrovascular aging on vascular cognitive impairment and dementia. Ageing Res Rev. 2017;34:15-29.

9. Choi JY, Morris JC, Hsu CY. Aging and cerebrovascular disease. Neurol Clin. 1998;16(3):687-711.

10. Marchant NL, Reed BR, DeCarli CS, Madison CM, Weiner MW, Chui HC et al. Cerebrovascular disease, beta-amyloid, and cognition in aging. Neurobiology of aging. 2012;33(5): 1006. e25-e36.

11. Mooradian AD. Potential mechanisms of the age-related changes in the blood-brain barrier. Neurobiol Aging. 1994;15(6):751-5. https://doi.org/10.1016/0197-4580(94 )90058-2.

12. Farrall AJ, Wardlaw JM. Blood-brain barrier: ageing and microvascular disease-systematic review and meta-analysis. Neurobiol Aging. 2009;30(3):337-52.

13. Varatharaj A, Galea I. The blood-brain barrier in systemic inflammation. Brain Behav Immun. 2017;60:1-12. https://doi.org/10.1016/j.bbi.2016.03.010.

14. Zenaro E, Piacentino G, Constantin G. The blood-brain barrier in Alzheimer's disease. Neurobiol Dis. 2017;107: 41-56. https://doi.org/10.1016/j.nbd.2016.07.007.

15. van de Haar HJ, Burgmans S, Jansen JF, van Osch MJ, van Buchem MA, Muller M, et al. Blood-brain barrier leakage in patients with early Alzheimer disease. Radiology. 2016;281(2):527-35.

16. Wang H, Golob EJ, Su M-Y. Vascular volume and bloodbrain barrier permeability measured by dynamic contrast enhanced MRI in hippocampus and cerebellum of patients with $\mathrm{MCI}$ and normal controls. J Magn Reson Imaging. 2006;24(3):695-700. https://doi.org/10.1002/jmri.20669.

17. Nation DA, Sweeney MD, Montagne A, Sagare AP, D'Orazio LM, Pachicano M, et al. Blood-brain barrier breakdown is an early biomarker of human cognitive dysfunction. Nat Med. 2019;25:270-6. https://doi.org/10.1038 /s41591-018-0297-y.

18. Zlokovic BV. Neurovascular pathways to neurodegeneration in Alzheimer\&\#39;s disease and other disorders. Nat Rev Neurosci. 2011;12:723. https://doi.org/10.1038 /nrn3114.

19. Wilhelm I, Nyúl-Tóth Á, Suciu M, Hermenean A, Krizbai IA. Heterogeneity of the blood-brain barrier. Tissue Barriers. 2016;4(1):e1143544. https://doi.org/10.1080 /21688370.2016.1143544.

20. Buxton RB, Uludağ K, Dubowitz DJ, Liu TT. Modeling the hemodynamic response to brain activation. Neuroimage. 2004;23:S220-S33.

21. Guo S, Lo EH. Dysfunctional cell-cell signaling in the neurovascular unit as a paradigm for central nervous system disease. Stroke. 2009;40(3_suppl_1):S4-7.

22. Zhao Z, Nelson AR, Betsholtz C, Zlokovic BV. Establishment and dysfunction of the blood-brain barrier. Cell. 2015;163(5):1064-78.

23. Pardridge WM. Blood-brain barrier endogenous transporters as therapeutic targets: a new model for small molecule CNS drug discovery. Expert Opin Ther Targets. 2015;19(8):1059-72. 
24. Montagne A, Zhao Z, Zlokovic BV. Alzheimer's disease: a matter of blood-brain barrier dysfunction? J Exp Med. 2017;214(11):3151-69.

25. Zlokovic BV. The blood-brain barrier in health and chronic neurodegenerative disorders. Neuron. 2008;57(2):178-201.

26. Wardlaw J, Sandercock P, Dennis M, Starr J. Is breakdown of the blood-brain barrier responsible for lacunar stroke, leukoaraiosis, and dementia? Stroke. 2003;34(3):806-12.

27. Zlokovic BV. Neurovascular mechanisms of Alzheimer's neurodegeneration. Trends Neurosci. 2005;28(4):202-8. https://doi.org/10.1016/j.tins.2005.02.001.

28. Kisler K, Nelson AR, Montagne A, Zlokovic BV. Cerebral blood flow regulation and neurovascular dysfunction in Alzheimer disease. Nat Rev Neurosci. 2017;18:419. https://doi.org/10.1038/nrn.2017.48.

29. Iadecola $C$. The neurovascular unit coming of age: a journey through neurovascular coupling in health and disease. Neuron. 2017;96(1):17-42. https://doi.org/10.1016/j. neuron.2017.07.030.

30. Csiszar A, Tarantini S, Yabluchanskiy A, Balasubramanian P, Kiss T, Farkas E, et al. Role of endothelial NAD+ deficiency in age-related vascular dysfunction. Am J Phys Heart Circ Phys. 2019;316(6):H1253-H66.

31. Raja R, Rosenberg GA, Caprihan A. MRI measurements of blood-brain barrier function in dementia: a review of recent studies. Neuropharmacology. 2018;134:259-71. https://doi. org/10.1016/j.neuropharm.2017.10.034.

32. Fernando MS, Simpson JE, Matthews F, Brayne C, Lewis $\mathrm{CE}$, Barber R, et al. White matter lesions in an unselected cohort of the elderly. Stroke. 2006;37(6):1391-8.

33. Burgmans S, van de Haar HJ, Verhey FR, Backes WH. Amyloid- $\beta$ interacts with blood-brain barrier function in dementia: a systematic review. J Alzheimers Dis. 2013;35(4):859-73.

34. Iadecola C, Zhang F, Niwa K, Eckman C, Turner SK, Fischer E, et al. SOD1 rescues cerebral endothelial dysfunction in mice overexpressing amyloid precursor protein. Nat Neurosci. 1999;2:157. https://doi.org/10.1038/5715.

35. Niwa K, Kazama K, Younkin L, Younkin SG, Carlson GA, Iadecola C. Cerebrovascular autoregulation is profoundly impaired in mice overexpressing amyloid precursor protein. Am J Phys Heart Circ Phys. 2002;283(1):H315-H23. https://doi.org/10.1152/ajpheart.00022.2002.

36. Niwa K, Kazama K, Younkin SG, Carlson GA, Iadecola C. Alterations in cerebral blood flow and glucose utilization in mice overexpressing the amyloid precursor protein. Neurobiol Dis. 2002;9(1):61-8. https://doi.org/10.1006 /nbdi.2001.0460.

37. Iturria-Medina $\mathrm{Y}$, Sotero RC, Toussaint PJ, Mateos-Pérez JM, Evans AC, The Alzheimer's Disease Neuroimaging I et al. Early role of vascular dysregulation on late-onset Alzheimer's disease based on multifactorial data-driven analysis. Nature Communications 2016;7:11934. doi: https://doi.org/10.1038/ncomms11934 https://www.nature. com/articles/ncomms11934\#supplementary-information.

38. Montagne A, Barnes SR, Sweeney MD, Halliday MR, Sagare AP, Zhao Z, et al. Blood-brain barrier breakdown in the aging human hippocampus. Neuron. 2015;85(2):296302.

39. Montagne A, Nation DA, Pa J, Sweeney MD, Toga AW, Zlokovic BV. Brain imaging of neurovascular dysfunction in Alzheimer's disease. Acta Neuropathol. 2016;131(5): 687-707.

40. Yamazaki Y, Zhao N, Caulfield TR, Liu C-C, Bu G. Apolipoprotein E and Alzheimer disease: pathobiology and targeting strategies. Nat Rev Neurol. 2019;15(9):50118. https://doi.org/10.1038/s41582-019-0228-7.

41. Bell RD, Winkler EA, Singh I, Sagare AP, Deane R, Wu Z, et al. Apolipoprotein E controls cerebrovascular integrity via cyclophilin A. Nature. 2012;485(7399):512-6. https://doi. org/10.1038/nature11087.

42. Salloway S, Gur T, Berzin T, Tavares R, Zipser B, Correia S, et al. Effect of APOE genotype on microvascular basement membrane in Alzheimer's disease. J Neurol Sci. 2002;203204:183-7. https://doi.org/10.1016/s0022-510x(02)002885.

43. Halliday MR, Rege SV, Ma Q, Zhao Z, Miller CA, Winkler EA, et al. Accelerated pericyte degeneration and blood-brain barrier breakdown in apolipoprotein E4 carriers with Alzheimer's disease. J Cereb Blood Flow Metab. 2016;36(1):216-27. https://doi.org/10.1038/jcbfm.2015.44.

44. Folstein MF, Folstein SE, McHugh PR. "Mini-mental state": a practical method for grading the cognitive state of patients for the clinician. J Psychiatr Res. 1975;12(3):189-98.

45. Montagne A, Nation DA, Sagare AP, Barisano G, Sweeney MD, Chakhoyan A, et al. APOE4 leads to blood-brain barrier dysfunction predicting cognitive decline. Nature. 2020;581(7806):71-6. https://doi.org/10.1038/s41586-0202247-3.

46. Larsson HB, Courivaud F, Rostrup E, Hansen AE. Measurement of brain perfusion, blood volume, and blood-brain barrier permeability, using dynamic contrastenhanced T1-weighted MRI at 3 tesla. Magnetic Resonance in Medicine: An Official Journal of the International Society for Magnetic Resonance in Medicine. 2009;62(5):1270-81.

47. van de Haar HJ, Burgmans S, Hofman PA, Verhey FR, Jansen JF, Backes WH. Blood-brain barrier impairment in dementia: current and future in vivo assessments. Neurosci Biobehav Rev. 2014.

48. Zhang CE, Wong SM, van de Haar HJ, Staals J, Jansen JF, Jeukens CR, et al. Blood-brain barrier leakage is more widespread in patients with cerebral small vessel disease. Neurology. 2017;88(5):426-32.

49. Pantoni L. Cerebral small vessel disease: from pathogenesis and clinical characteristics to therapeutic challenges. The Lancet Neurology. 2010;9(7):689-701.

50. Verheggen IC, de Jong JJ, van Boxtel MP, Gronenschild EH, Palm WM, Postma AA, et al. Increase in blood-brain barrier leakage in healthy, older adults. GeroScience. 2020: $1-11$.

51. Jolles J, Houx P, Van Boxtel M, Ponds R. Maastricht aging study: determinants of cognitive aging. Neuropsych Publishers Maastricht; 1995. 
52. Gélinas I, Gauthier L, McIntyre M, Gauthier S. Development of a functional measure for persons with Alzheimer's disease: the disability assessment for dementia. Am J Occup Ther. 1999;53(5):471-81.

53. Rey A. L'examen clinique en psychologie. 1958.

54. Natu M, Agarwal A. Digit letter substitution test (DLST) as an alternative to digit symbol substitution test (DSST). Hum Psychopharmacol Clin Exp. 1995;10(4):339-43.

55. Stroop JR. Studies of interference in serial verbal reactions. J Exp Psychol. 1935;18(6):643.

56. Fazekas F, Kleinert R, Offenbacher H, Schmidt R, Kleinert G, Payer F, et al. Pathologic correlates of incidental MRI white matter signal hyperintensities. Neurology. 1993;43(9): 1683.

57. Pasquier F, Leys D, Weerts JG, Mounier-Vehier F, Barkhof F, Scheltens P. Inter-and intraobserver reproducibility of cerebral atrophy assessment on MRI scans with hemispheric infarcts. Eur Neurol. 1996;36(5):268-72.

58. Scheltens P, Leys D, Barkhof F, Huglo D, Weinstein H, Vermersch P, et al. Atrophy of medial temporal lobes on MRI in "probable" Alzheimer's disease and normal ageing: diagnostic value and neuropsychological correlates. J Neurol Neurosurg Psychiatry. 1992;55(10):967-72.

59. Jacobs HI, Clerx L, Gronenschild EH, Aalten P, Verhey FR. White matter hyperintensities are positively associated with cortical thickness in Alzheimer's disease. J Alzheimers Dis. 2014;39(2):409-22.

60. Schwarz CG, Gunter JL, Wiste HJ, Przybelski SA, Weigand $\mathrm{SD}$, Ward CP, et al. A large-scale comparison of cortical thickness and volume methods for measuring Alzheimer's disease severity. NeuroImage: Clinical. 2016;11:802-12. https://doi.org/10.1016/j.nicl.2016.05.017.

61. Fischl B, Sereno MI, Dale AM. Cortical surface-based analysis: II: inflation, flattening, and a surface-based coordinate system. NeuroImage. 1999;9(2):195-207. https://doi. org/10.1006/nimg.1998.0396.

62. van de Haar HJ, Jansen JF, van Osch MJ, van Buchem MA, Muller M, Wong SM, et al. Neurovascular unit impairment in early Alzheimer's disease measured with magnetic resonance imaging. Neurobiol Aging. 2016;45:190-6.

63. Cramer SP, Larsson HB. Accurate determination of bloodbrain barrier permeability using dynamic contrast-enhanced T1-weighted MRI: a simulation and in vivo study on healthy subjects and multiple sclerosis patients. J Cereb Blood Flow Metab. 2014;34(10):1655-65.

64. Faul F, Erdfelder E, Lang A-G, Buchner A. G* Power 3: a flexible statistical power analysis program for the social, behavioral, and biomedical sciences. Behav Res Methods. 2007;39(2):175-91.

65. Cohen J. A power primer. Psychol Bull. 1992;112(1):155.

66. Faul F, Erdfelder E, Buchner A, Lang A-G. Statistical power analyses using $\mathrm{G}^{*}$ Power 3.1: tests for correlation and regression analyses. Behav Res Methods. 2009;41(4):114960.

67. Benjamini Y, Hochberg Y. Controlling the false discovery rate: a practical and powerful approach to multiple testing. $\mathrm{J}$ R Stat Soc Ser B Methodol. 1995;57(1):289-300.
68. Van Der Elst W, Van Boxtel MP, Van Breukelen GJ, Jolles J. Rey's verbal learning test: normative data for 1855 healthy participants aged 24-81 years and the influence of age, sex, education, and mode of presentation. J Int Neuropsychol Soc. 2005; 11(03):290-302.

69. Harada CN, Love MCN, Triebel KL. Normal cognitive aging. Clin Geriatr Med. 2013;29(4):737-52.

70. Rönnlund M, Nyberg L, Bäckman L, Nilsson L-G. Stability, growth, and decline in adult life span development of declarative memory: cross-sectional and longitudinal data from a population-based study. Psychol Aging. 2005;20(1):3-18. https://doi.org/10.1037/0882-7974.20.1.3.

71. Eichenbaum H. Hippocampus: cognitive processes and neural representations that underlie declarative memory. Neuron. 2004;44(1):109-20.

72. Eichenbaum H. A cortical-hippocampal system for declarative memory. Nat Rev Neurosci. 2000;1(1):41-50.

73. Freeze WM, Jacobs HI, de Jong JJ, Verheggen IC, Gronenschild EH, Palm WM, et al. White matter hyperintensities mediate the association between bloodbrain barrier leakage and information processing speed. Neurobiol Aging. 2020;85:113-22.

74. Salthouse TA. When does age-related cognitive decline begin? Neurobiol Aging. 2009;30(4):507-14.

75. Machulda MM, Pankratz VS, Christianson TJ, Ivnik RJ, Mielke MM, Roberts RO, et al. Practice effects and longitudinal cognitive change in normal aging vs. incident mild cognitive impairment and dementia in the Mayo Clinic Study of Aging. Clin Neuropsychol. 2013;27(8):1247-64.

76. McCaffrey RJ, Ortega A, Haase RF. Effects of repeated neuropsychological assessments. Arch Clin Neuropsychol. 1993;8(6):519-24.

77. Salthouse TA. Selective review of cognitive aging. J Int Neuropsychol Soc. 2010;16(5):754-60.

78. Wecker NS, Kramer JH, Wisniewski A, Delis DC, Kaplan E. Age effects on executive ability. Neuropsychology. 2000;14(3):409.

79. Daigneault S, Braun CM, Whitaker HA. Early effects of normal aging on perseverative and non-perseverative prefrontal measures. Dev Neuropsychol. 1992;8(1):99-114.

80. Charlton RA, Barrick TR, Lawes INC, Markus HS, Morris RG. White matter pathways associated with working memory in normal aging. Cortex. 2010;46(4):474-89.

81. Charlton RA, Barrick TR, McIntyre DJ, Shen Y, O'Sullivan $\mathrm{M}$, Howe FA, et al. White matter damage on diffusion tensor imaging correlates with age-related cognitive decline. Neurology. 2006;66(2):217-22. https://doi.org/10.1212/01. wnl.0000194256.15247.83.

82. Constantinidis C, Procyk E. The primate working memory networks. Cognitive, Affective, \& Behavioral Neuroscience. 2004;4(4):444-65. https://doi.org/10.3758/CABN.4.4.444.

83. Daigneault S, Braun CM. Working memory and the selfordered pointing task: further evidence of early prefrontal decline in normal aging. J Clin Exp Neuropsychol. 1993;15(6):881-95.

84. Lista I, Sorrentino G. Biological mechanisms of physical activity in preventing cognitive decline. Cell Mol Neurobiol. 2010;30(4):493-503. 
85. Ajmani R, Metter E, Jaykumar R, Ingram D, Spangler E, Abugo $\mathrm{O}$, et al. Hemodynamic changes during aging associated with cerebral blood flow and impaired cognitive function. Neurobiol Aging. 2000;21(2):257-69.

86. Kazakos EI, Kountouras J, Polyzos SA, Deretzi G. Novel aspects of defensins' involvement in virus-induced autoimmunity in the central nervous system. Medical Hypotheses. 2017;102(Supplement C):33-6. https://doi.org/10.1016/j. mehy.2017.02.020.

87. Li Y, Li M, Zuo L, Shi Q, Qin W, Yang L, et al. Compromised blood-brain barrier integrity is associated with total magnetic resonance imaging burden of cerebral small vessel disease. Front Neurol. 2018;9:221. https://doi. org/10.3389/fneur.2018.00221.

88. Shlosberg D, Benifla M, Kaufer D, Friedman A. Bloodbrain barrier breakdown as a therapeutic target in traumatic brain injury. Nat Rev Neurol. 2010;6(7):393-403.

89. Debette S, Beiser A, DeCarli C, Au R, Himali JJ, KellyHayes M, et al. Association of MRI markers of vascular brain injury with incident stroke, mild cognitive impairment, dementia, and mortality: the Framingham Offspring Study.
Stroke. 2010;41(4):600-6. https://doi.org/10.1161 /strokeaha.109.570044.

90. Shapiro DM, Harrison DW. Alternate forms of the AVLT: a procedure and test of form equivalency. Arch Clin Neuropsychol. 1990;5(4):405-10.

91. Mills KF, Yoshida S, Stein LR, Grozio A, Kubota S, Sasaki $\mathrm{Y}$, et al. Long-term administration of nicotinamide mononucleotide mitigates age-associated physiological decline in mice. Cell Metab. 2016;24(6):795-806.

92. Zhang H, Ryu D, Wu Y, Gariani K, Wang X, Luan P, et al. $\mathrm{NAD}+$ repletion improves mitochondrial and stem cell function and enhances life span in mice. Science. 2016;352(6292):1436-43.

93. Yoshino J, Baur JA. Imai S-i. NAD+ intermediates: the biology and therapeutic potential of NMN and NR. Cell Metab. 2018;27(3):513-28.

Publisher's note Springer Nature remains neutral with regard to jurisdictional claims in published maps and institutional affiliations. 